Bruno Lopes BASTOS ${ }^{1}$

Gerson de Oliveira

NORBERTO ${ }^{1}$

Rodrigo MAIA-NOGUEIRA ${ }^{1}$

José Eugênio GUIMARÃES²

Correspondência para:

BRUNOLOPESBASTOS

Largo da Vitória, 02/102, Ed. San Remo

40130-110-Salvador-BA

bbastos@pop.com.br

Recebido para publicação: 26/01/2004 Aprovado para publicação: 01/06/2005

\title{
Avaliação hematológica e dosagem bioquímica de ALT, AST e creatinina em elefante-marinho-do-sul, Mirounga leonina (linnaeus, 1758), encontrado no litoral de Salvador, Bahia
}

\author{
1 - Centro de Resgate de Mamíferos Aquáticos - CRMA / Sociedade de Pesquisa \\ e Conservação dos Mamíferos Aquáticos. \\ 2 - Laboratório de Análises Clínicas - LAC do Departamento de Patologia e \\ Clínicas da Escola de Medicina Veterinária da Universidade Federal da Bahia - \\ Salvador - BA
}

\section{Resumo}

O Centro de Resgate de Mamíferos Aquáticos (CRMA) tem trabalhado desde 1999 com o resgate e a reabilitação de cetáceos e pinípedes em situação de encalhe no litoral do Estado da Bahia. O presente trabalho objetiva apresentar e avaliar os resultados de hemogramas e dosagens séricas de alanina aminotranferase (ALT), aspartato aminotransferase (AST) e creatinina realizados em um exemplar de elefante-marinhodo-sul, Mirounga leonina (LINNAEUS, 1758), encontrado no dia 11 de fevereiro de 2002 na praia da Barra, litoral de Salvador, BA. Tratavase de um filhote macho, com $137 \mathrm{~cm}$ de comprimento e peso aproximado de $49 \mathrm{~kg}$, com estado nutricional debilitado, um ferimento no lado direito na altura da escápula. $\mathrm{O}$ exemplar permaneceu em acompanhamento clínico por 56 dias, sendo vermifugado com Febendazole, o ferimento suturado e tratado com álcool iodado, Nitrofurazona solução e Tartarato de Ketanserina, simultaneamente com Enrofloxacina 10\%, Potenay ${ }^{\circledR}$, Complexo B e Benerva ${ }^{\circledR}$. No dia 16 seguinte apresentou um quadro de conjuntivite unilateral direita, sendo tratado até o final de sua estadia com pomada oftálmica à base de Cloridrato de Cloranfenicol. Durante este período colheu-se um total de seis amostras de sangue, sendo três para a realização de hemograma e as demais para dosagem de ALT, AST e creatinina. De acordo com os resultados dos eritrogramas o filhote desenvolveu um quadro de anemia, classificada como microcítica e normocrômica. Com relação aos leucogramas, destacou-se linfopenia, eosinopenia e monocitopenia, possivelmente influenciado pelas condições de estresse e o manejo. Constatou-se nos exames bioquímicos valores diminuídos para a AST e creatinina, porém não representando a existência de um quadro patológico de significância clínica.
\end{abstract}

\section{Introdução}

O elefante-marinho-do-sul, Mirounga leonina (LINNAEUS, 1758), é uma espécie circumpolar ocorrendo naturalmente nas ilhas sub-antárticas dos oceanos Atlântico, Índico e Pacífico, onde se reproduz ${ }^{1}$. $\mathrm{Na}$ América do Sul, possui ocorrência natural na península Valdez (Argentina) e na ilha Juan
Palavras-chave: Elefante-marinho-dosul.

Mirounga leonina. Hemograma. Bioquímica sérica. Anemia.
Fernandez (Chile), não existindo colônias reprodutivas em nosso litoral, sendo os poucos registros presentes representados por machos e indivíduos jovens ${ }^{2}$.

Desde 1999, o Centro de Resgate de Mamíferos Aquáticos (CRMA) tem trabalhado com o resgate e a reabilitação de cetáceos e pinípedes em situação de encalhe no litoral do Estado da Bahia. Dentro deste 
contexto, exames laboratoriais como o hemograma e bioquímico de algumas enzimas constituem uma importante ferramenta para o diagnóstico de enfermidades nestes animais, podendo ser detectadas alterações fisiológicas momentâneas ${ }^{3,4,5}$, infecções agudas ou crônicas ${ }^{6,7,8}$, bem como processos neoplásicos ${ }^{9}$.

Este trabalho tem como objetivo apresentar os resultados de hemogramas e dosagens séricas de alanina aminotranferase (ALT), aspartato aminotransferase (AST) e creatinina realizados em um exemplar de elefante-marinho-do-sul, Mirounga leonina, encontrado debilitado no litoral de Salvador, Bahia.

\section{Materiais e Métodos}

O animal (CRMA\#0006) foi encontrado no dia 11 de fevereiro de 2002 na praia do

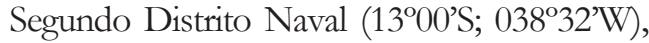
Barra, Salvador, Bahia. Após o seu resgate, realizou-se exame clínico constatando-se tratar de um filhote macho, com $137 \mathrm{~cm}$ de comprimento e peso aproximado de $49 \mathrm{~kg}$, com estado nutricional debilitado, um ferimento no lado direito na altura da escápula e temperatura de $40,8^{\circ} \mathrm{C}$.

O filhote foi acondicionado em sala com ar condicionado, resfriado com toalhas molhadas e gelo em escamas, permanecendo em um tanque de $1,6 \mathrm{~m}^{3}$ de água doce por três dias. Em seguida foi transferido para uma piscina no Parque Zoobotânico Getúlio Vargas com dimensões adequadas para pinípedes segundo a Instrução Normativa $\mathrm{N}^{0} 3$ do IBAMA $^{10}$ de 13 de fevereiro de 2002, a qual recebeu manutenção sistemática a cada $72 \mathrm{~h}$, com desinfecção com solução à base de Iodo e com varredura e flambagem. Os padrões da água foram mantidos em 250NMP de coliformes por $100 \mathrm{~mL} ; \mathrm{pH}$ entre 7,2 e 8,$0 ; 0,5 \mathrm{mg} / \mathrm{L}$ de cloro; e temperatura máxima de $28^{\circ} \mathrm{C}$.

$\mathrm{O}$ ferimento foi suturado e tratado com álcool iodado, Nitrofurazona solução e Tartarato de Ketanserina, simultaneamente com antibioticoterapia à base de Enrofloxacina
$10 \%$, com doses diárias de $3,5 \mathrm{~mL}$, intramuscular, por cinco dias, sendo sua cicatrização bem sucedida.

A dieta foi estabelecida em $6 \%$ do seu peso em presas, constituída de sardinhas, suplementos (Potenay ${ }^{\circledR}$, Complexo B e Benerva ${ }^{\circledR}$ ), sal marinho e, ocasionalmente, camarões como atrativo alimentar.

Através de exame parasitológico das fezes constatou-se a presença de ovos de parasitos da super-família Strongyloidea, sendo o exemplar vermifugado com Febendazole. Os exames posteriores apresentaram resultados negativos.

No dia 16 de fevereiro de 2002 o indivíduo apresentou um quadro de conjuntivite unilateral direita, sendo tratado até o final de sua estadia com pomada oftálmica à base de Cloridrato de Cloranfenicol. Observou-se uma melhora clínica a partir do $37^{\circ}$ dia após o início da conduta terapêutica, sendo adotada como medida profilática, a partir deste período, banhos de água salgada em um tanque alternativo de $4500 \mathrm{~L}$.

O exemplar permaneceu em acompanhamento clínico até o dia 08 de abril de 2002, sendo transferido na manhã deste mesmo dia para a cidade de Rio Grande (RS), onde seria submetido a um processo de readaptação ambiental para uma posterior soltura pelo Centro de Recuperação de Animais Marinhos (CRAM) e Núcleo de Educação e Monitoramento Ambiental (NEMA/IBAMA). Neste período, o elefante-marinho apresentava $185 \mathrm{~cm}$ de comprimento total e $85 \mathrm{~kg}$ de massa corporal.

Para a realização de exames laboratoriais complementares, colheu-se um total de seis amostras de $2,0 \mathrm{~mL}$ de sangue, através das veias interdigitais próximas à base dos membros posteriores do animal, sendo três acondicionadas em tubos contendo EDTA, para a realização de hemograma, e as demais sem anticoagulante para obtenção de soro para as dosagens de alanina aminotranferase (ALT), aspartato aminotransferase (AST) e creatinina. 
O material colhido foi enviado imediatamente ao laboratório para análise, sendo os hemogramas realizados pelo método de contagem manual em câmara de Neubauer, e as dosagens bioquímicas das transaminases e creatinina pelos métodos de Reitman / Frankel e Jaffé modificado, respectivamente. Após a constatação de alteração nas variáveis analisadas no eritrograma, realizou-se o cálculo dos índices hematimétricos para a classificação da anemia.

Os dados obtidos nos hemogramas e dosagens séricas de ALT, AST e creatinina foram analisados e comparados com valores de referência de elefantes-marinhos-do-sul e do-norte, Mirounga leonina e M. angustirostris, respectivamente.

\section{Resultados e Discussão}

Os resultados dos hemogramas encontram-se na tabela 1. Observa-se no Exame H1 que os valores médios das variáveis que compõem o eritrograma; número de hemácias, concentração de hemoglobina e hematócrito encontram-se dentro ou bem próximos das amplitudes citadas por Lane, Morris e Sheedy ${ }^{4}$ e Lewis, Uhart e Ortiz ${ }^{5}$ (Figura 1). Segundo os primeiros autores o volume globular médio (VGM) e a hemoglobina globular média (HGM) encontram-se abaixo do valor mínimo, enquanto a concentração de hemoglobina globular média (CHGM) apresenta-se dentro da amplitude, levando a uma discreta microcitose. Vale ressaltar que o animal em questão apresentava poucos meses de vida.

Considerando os exames $\mathrm{H} 2$ e H3, constata-se um comportamento bastante semelhante entre eles, porém diferente do primeiro exame, ocorrendo uma diminuição dos valores médios nas variáveis do eritrograma e um aumento do índice HGM, atingindo a normalidade no exame $\mathrm{H} 2$, levando a uma anemia do tipo microcítica normocrômica, conforme os valores citados por Lane, Morris e Sheedy ${ }^{4}$ e Lewis, Uhart e $\operatorname{Ortiz}^{5}$ (Figura 1). Possivelmente, esse quadro seja resultante de deficiência de ferro ou defeito na utilização dos estoques deste mineral conforme cita Jain?. O tempo de indução desta deficiência em filhotes é constituído apenas por semanas, de acordo com Bossart et al. ${ }^{7}$.

Com relação aos leucogramas, constata-se que os valores obtidos na contagem total de leucócitos encontram-se dentro da amplitude, exceto no exame $\mathrm{H} 2$ no qual se apresenta acima do valor máximo citado por Bryden e $\mathrm{Lim}^{3}$. No que se refere à contagem diferencial de leucócitos, quando comparada com os valores de referência de indivíduos da espécie Mirounga angustirostris, conforme citação de Bossart et al. ${ }^{7}$ (Figura 1), verifica-se apenas uma linfopenia no Exame H1, estando os demais tipos de leucócitos dentro das amplitudes. Já, no Exame $\mathrm{H} 2$ constata-se que a quantidade de basófilos, neutrófilos segmentados e linfócitos se mantêm dentro da normalidade, porém os monócitos encontram-se abaixo do valor mínimo de referência, fato este também ocorrido no Exame H3, demonstrando uma mono-citopenia. Contudo, no que se refere à quantidade de eosinófilos, no Exame H2 esta decresceu a limites muito abaixo do valor mínimo descrito, porém ultrapassando o valor máximo no último exame, determinando quadros de eosinopenia e eosinofilia, respectivamente, sendo o primeiro compatível com um estado de estresse.

$\mathrm{Na}$ tabela 2 destacam-se os resultados das dosagens bioquímicas de alanina aminotranferase (ALT), aspartato aminotransferase (AST) e creatinina. Observase nos exames B1, B2 e B3 que os valores da ALT encontram-se dentro da amplitude, enquanto que a AST esta abaixo do valor mínimo citado por Engelhard et al. . (Figura $^{8}$ 2) para filhotes de $M$. leonina. De acordo com estes autores, observa-se também que o resultado obtido no exame B1 para a creatinina está compatível com a normalidade, porém decrescendo nos exames seguintes para valores abaixo do mínimo descrito para a espécie.

Pelo fato da AST ser distribuída por 
Tabela 1 - Valores da contagem total de eritrócitos, determinação da concentração de hemoglobina, hematócrito, volume globular médio (VGM), hemoglobina globular média (HGM) e concentração de hemoglobina globular média (CHGM), e contagem total e diferencial absoluta de leucócitos de um filhote de elefante-marinho-do-sul, Mirounga leonina, nas datas 21/02/02, 26/03/02 e 03/04/02 (Exame H1, Exame $\mathrm{H} 2$ eExame H3, respectivamente) em Salvador., Bahia, 2002

\begin{tabular}{cccc}
\hline Parâmetro & Exame H1 & Exame $\mathrm{H} 2$ & Exame H3 \\
\hline Hemácias $(\mathrm{x}$ & 3,1 & 2,0 & 2,1 \\
$\left.10^{6} / \mathrm{mm}^{3}\right)$ & & & 12,5 \\
Hemoglobina $(\mathrm{g} / \mathrm{dL})$ & 18,9 & 13,0 & 38 \\
Hematócrito $(\%)$ & 54 & 35 & 181 \\
VGM $(\mathrm{fL})$ & 174 & 175 & 60 \\
HGM $(\mathrm{pg})$ & 61 & 65 & 33 \\
CHGM $(\%)$ & 35 & 37 & 19100 \\
Leucócitos $\left(/ \mathrm{mm}^{3}\right)$ & 14200 & 23500 & 0 \\
Basófilos $\left(/ \mathrm{mm}^{3}\right)$ & 0 & 0 & 1337 \\
Eosinófilos $\left(/ \mathrm{mm}^{3}\right)$ & 142 & 0 & 0 \\
Bastonetes $\left(/ \mathrm{mm}^{3}\right)$ & 0 & 0 & 12797 \\
Neutrófilos & 10366 & 16685 & 4966 \\
segmentados $\left(/ \mathrm{mm}^{3}\right)$ & 1278 & 6815 & 0 \\
Linfócitos $\left(/ \mathrm{mm}^{3}\right)$ & 2414 & 0 & \\
Monócitos $\left(/ \mathrm{mm}^{3}\right)$ & & & \\
\hline
\end{tabular}

\begin{tabular}{|c|c|c|c|c|}
\hline Parâmetro & $\begin{array}{l}\text { LANE et al. } \\
\quad(1972)\end{array}$ & $\begin{array}{l}\text { LEWIS } \\
(2001)\end{array}$ & $\begin{array}{c}\text { BRYDEN \& LIM } \\
(1969)\end{array}$ & $\begin{array}{c}\text { BOSSART et al. } \\
(2001)\end{array}$ \\
\hline Hemácias $\left(\times 10^{6} / \mathrm{mm}^{3}\right)$ & $2,75 \pm 0,20 *$ & $2,6 \pm 0,4^{*}$ & -- & -- \\
\hline Hemoglobina (g / dL) & $20,7 \pm 1,84^{*}$ & $19,8 \pm 2,3^{*}$ & --. & --- \\
\hline Hematócrito (\%) & $54,8 \pm 3,68^{*}$ & $54,4 \pm 4,5^{*}$ & --- & - \\
\hline VGM (fL) & $197 \pm 14,32 *$ & --.-- & -- & - \\
\hline HGM (pg) & $72,4 \pm 7,50^{*}$ & --- & --- & --- \\
\hline $\mathrm{CHGM}(\%)$ & $37,6 \pm 4,34^{*}$ & - & - & - \\
\hline Leucócitos $\left(/ \mathrm{mm}^{3}\right)$ & - & - & $15240 \pm 4380^{*}$ & $10600-28500$ \\
\hline Basófilos $\left(/ \mathrm{mm}^{3}\right)$ & - & - & - & $0-160$ \\
\hline Eosinófilos (/mm³) & - & - & - & $100-795$ \\
\hline Bastonetes $\left(/ \mathrm{mm}^{3}\right)$ & - & --.- & - & $0-527$ \\
\hline Neutrófilos segmentados $\left(/ \mathrm{mm}^{3}\right)$ & - & - & - & $7176-20714$ \\
\hline Linfócitos $\left(/ \mathrm{mm}^{3}\right)$ & --.-- & -- & --- & $2385-7630$ \\
\hline Monócitos (/mm³) & --- & ---- & --- & $400-3400$ \\
\hline
\end{tabular}

*Média \pm Desvio Padrão

Figura 1 - Valores de eritrócitos, hemoglobina, hematócrito, volume globular médio (VGM), hemoglobina globular média (HGM), concentração de hemoglobina globular média (CHGM) e contagem total de leucócitos de filhotes de elefante-marinho-do-sul, Mirounga leonina, e contagem total e diferencial absoluta de leucócitos de elefantes-marinhos-do-norte juvenis, Mirounga angustirostris 
Tabela 2 - Valores séricos de alanina aminotranferase (ALT), aspartato aminotransferase (AST), glicose ecreatinina de um filhote de elefante-marinhodo-sul, Mirounga leonina, nas datas 21/02/02,02/03/02 e 25/03/02 (Exame B1, Exame B2 e Exame B3 respectivamente) em Salvador, Bahia, 2002.

\begin{tabular}{cccc}
\hline Parâmetro & Exame B3 & Exame B1 & Exame B2 \\
\hline ALT $(\mathrm{U} / \mathrm{I})$ & 33 & 22 & 13,4 \\
AST $(\mathrm{U} / \mathrm{I})$ & 21 & 48 & 26 \\
Creatinina $(\mathrm{mg} / \mathrm{dL})$ & $1,1(97,2)^{*}$ & $0,6(53,0)^{*}$ & $0,8(70,7)^{*}$ \\
\hline
\end{tabular}

$*$ ou $\mu \mathrm{mol} / \mathrm{L}$

\begin{tabular}{cc}
\hline Parâmetro & Engelhardet al.(2002) \\
\hline ALT (U/l) & $28 \pm 17^{*}$ \\
AST (U/I) & $67 \pm 14^{*}$ \\
Creatinina $(\mu \mathrm{mol} / \mathrm{l})$ & $86 \pm 13^{*}$ \\
\hline
\end{tabular}

Figura 2 - Valores bioquímicos (Média \pm Desvio Padrão) de alanina aminotranferase (ALT), aspartato aminotransferase (AST) e creatinina de filhotes de elefante-marinho-do-sul, Mirounga leonina, durante o estágio final do seu período de lactação

vários tecidos do corpo dos pinípedes, e não sendo recomendada individualmente para avaliação da função hepática ${ }^{6}$, a alteração constatada não pode ser considerada um indicador de lesão hepática. Com relação à creatinina, possivelmente a diminuição dos seus valores ocorreu devido a uma redução na massa muscular e um aumento da concentração de colesterol sérico e da camada de gordura, modificações morfofisiológicas que ocorrem no final do período de lactação destes animais ${ }^{3}$.

\section{Conclusão}

Frente aos resultados dos hemo-gramas, pode-se concluir que o presente elefantemarinho-do-sul desenvolveu um quadro de anemia, classificada como microcítica normocrômica. As condições de estresse e o manejo possivelmente contribuiram para o quadro leucocitário instável, destacando-se linfopenia, eosino-penia e monocitopenia.

As alterações constatadas nas dosagens bioquímicas não se apresentam como indicadores clássicos de um quadro patológico, estando possivelmente envolvidas com modificações morfofisiológicas que ocorrem nesta fase de vida destes animais. Considerando-se a diversidade de fatores que possam influenciar o extravasamento das enzimas hepáticas e renal, supõe-se que muito provavelmente o estresse esteja atuando como uma variável preponderante sobre este animal, porém não sendo de tal magnitude a ponto de atuar sobre os órgãos/tecidos responsáveis pela sua liberação na corrente sangüínea, comprovando desta maneira o seu não envolvimento nos valores das enzimas aqui analisadas.

\section{Agradecimentos}

A todos os membros do Centro de Resgate de Mamíferos Aquáticos (CRMA) e demais unidades da Sociedade de Pesquisa e Conservação dos Mamíferos Aquáticos. Agradecemos também ao Parque Zoobotânico Getúlio Vargas (Zoo Salvador), CITVET II Centro de Diagnóstico Veterinário e Dra. Márcia Hungar, e aos consultores anônimos pela revisão e sugestões ao manuscrito. 


\title{
Haematological analysis and clinical chemistry of ALT, AST and creatinine of a southern elephant seal, Mirounga leonina (linnaeus, 1758), found on the Salvador coast, Bahia
}

\begin{abstract}
Since 1999 the Aquatic Mammals Rescue Center - AMRC has been working in the rescue and rehabilitation of stranded cetaceans and pinnipeds on the coast of Bahia, Brazil. This paper presents and analyses the blood cells count and clinical chemistry of alanine aminotransferase (ALT), aspartate aminotransferase (AST) and creatinine of a southern elephant seal, Mirounga leonina (LINNAEUS, 1758), found on February the $11^{\text {th }}$ at Barra Beach, Salvador, BA. The specimen was an orphan male calf, with $137 \mathrm{~cm}$ of length and estimated weight of $49 \mathrm{~kg}$. It presented bad nutritional conditions and a shark bite on the right shoulder area. Clinical management was performed for 56 days, anthelmintic Febendazole was utilized, and the bite was treated with iodined alcohol, Nitrofurazone solution and Kethanserin, simultaneously with Enrofloxacin 10\%, Potenay ${ }^{\circledR}$, Vitamin B Complex and Benerva ${ }^{\circledR}$. On the $16^{\text {th }}$ the animal presented a right unilateral conjuntivitis, treated with Cloranphenicol oftalmic pomade until the end of its stay in the captive. During this period a total of six blood samples were collected, three for total blood counts and the others for the biochemistry determination of ALT, AST and creatinine. According to the haematological analysis the seal developed an anaemia which was classified as microcytic and normochromic. Lymphopenia, eosinopenia and monocytopenia were also observed, possibly due to its handling and stress conditions. The clinical chemistry presented low values for AST and creatinine, although this did not represent the existence of any pathologic context or disease with clinical significance.
\end{abstract}

Key words:

\section{Referências}

1 JEFFERSON, T. A.; LEATHERWOOD, S.; WEBBER, M. A. Marine mammals of the world: FAO species identification guide. Roma: UNEP/FAO, 1993. p. 286 $-287$.

2 IBAMA. Mamíferos aquáticos do Brasil: plano de ação. Brasília: Instituto Brasileiro do Meio Ambiente e dos Recursos Naturais Renováveis, 2001. 96 p.

3 BRYDEN, M. M.; LIM, G. H. K. Blood parameters of the southern elephant seal (Mirounga leonina, LINN.) in relation to diving. Comparative Biochemistry and Physiology, v. 28, 1969. p. 139-148, 1969.

4 LANE, R. A. B.; MORRIS, R. J. H.; SHEEDY, J. W. A haematological study of the southern elephant seal, Mirounga leonina (LINN.). Comparative Biochemistry and Physiology. v. 42A, p. 841-850, 1972.

5 LEWIS, M.; UHART, M.; ORTIZ, C. L. Ontogenetic and seasonal variation in blood parameters in southern elephant seals. Marine Mammals Science, v. 17, n. 4, p. 862-872, 2001.
6 MEDWAY, W.; GERACI, J. R. Clinical pathology of marine mammals. In: FOWLER, M. E. Zoo \& wild animal medicine. 2. ed. Philadelphia: W. B. Saunders, 1986. p. 791-797.

7 BOSSART, G. D.; REIDARSON, T.H.; DIERAUF, L.A.; DUFFIELD, D.A. Clinical Pathology. In: DIERAUF, L. A. \& GULLAND, F. M. D. CRC Handbook of marine mammal medicine, 2. ed., New York: CRC Press.2001. p. $383-436$.

8 ENGELHARD, G. H.; HALL, A.J.; BRASSEUR, S.M.J.M.; REIJNDERS, P.J.H. Blood chemistry in southern elephant seal mothers and pups during lactation reveals no effect of handling. Comparative Biochemistry and Physiology, Part A, n. 133, 367-378, 2002.

9 JAIN, N. C. Essentials of veterinary hematology. Philadelphia: Lea \& Febiger, 1993. 417 p.

10 IBAMA. Instrução Normativa No $\mathbf{3}$, de $\mathbf{1 3}$ de fevereiro de 2002. Brasília: Instituto Brasileiro do Meio Ambiente e dos Recursos Naturais Renováveis, 2002. $13 \mathrm{p}$. 